\title{
Superficial plane endoscopy for carpal tunnel release
}

\author{
Bartłomiej H. Noszczyk, Natalia Krzesniak, Marcin Nowak \\ Medical Centre of Postgraduate Education, Warsaw, Poland
}

Videosurgery Miniinv 2014; 9 (2): 262-266

DOI: $10.5114 /$ wiitm.2014.41632

\begin{abstract}
In this publication we describe three techniques for endoscopic carpal tunnel release which use the palmar portal approach and the superficial plane to enable a view from above the transverse carpal ligament. These techniques prevent any iatrogenic compression of the median nerve inside the already narrow tunnel. Although the necessary instruments are inexpensive and recommended in instrumentation catalogues, their use has rarely been reported. These techniques are safe and easy to learn for surgeons familiar with arthroscopy. We believe that they constitute an important alternative to the already well-established methods of interior tunnel access, and believe that their use should become more widespread.
\end{abstract}

Key words: carpal tunnel syndrome, carpal tunnel release, endoscopic techniques, endoscopic retractor, endoscope guide.

\section{Introduction}

The majority of endoscopic techniques for carpal tunnel release use endoscopes equipped with trocars, or blades, to cut the transverse carpal ligament (TCL) from its undersurface [1]. Fewer techniques exist that rely on instruments that guide the endoscope and blade from outside the carpal tunnel, enabling a view from above.

We describe three such techniques, two of which use retractors, and one which uses a tube guide. These techniques prevent the median nerve from being put under excessive pressure inside the already narrow tunnel. They are easy to learn for surgeons familiar with arthroscopy. Our initial results from several hundred such releases were better than those using standard mini-incision techniques [2].

\section{Technical notes}

A 7-10 $\mathrm{mm}$ incision is created distally from the line connecting the ulnar side of the thumb and hamate (modified Kaplan's line), and collaterally to the ulnar side of the thenar crease. The subcutaneous fat and fibres of the thenar aponeurosis are drawn aside to access the palmar fat pad, a landmark during carpal tunnel release. The distal end of the TCL can be found immediately proximally to the palmar fat pad, at the proximal border of the skin incision. A superficial endoscopic carpal tunnel release requires that the guide, or retractor with an integrated endoscope, be inserted under the skin or between the superficial and deeper TCL surface. We use three such instruments: the Medicon endoscope guide (Medicon eG, Tuttlingen, Germany), the Krishnan optical retractor (Karl Storz GmbH, Tuttlingen, Germany), and the endoscope sheath with distal beak for restraining tissue (Karl Storz GmbH, Tuttlingen, Germany) (Photo 1).

The Medicon guide has a blunt tip, and a longitudinal slit at the bottom. The guide should be pushed proximally into the subcutaneous tissue or under the superficial TCL layer, without any dissection. Care 


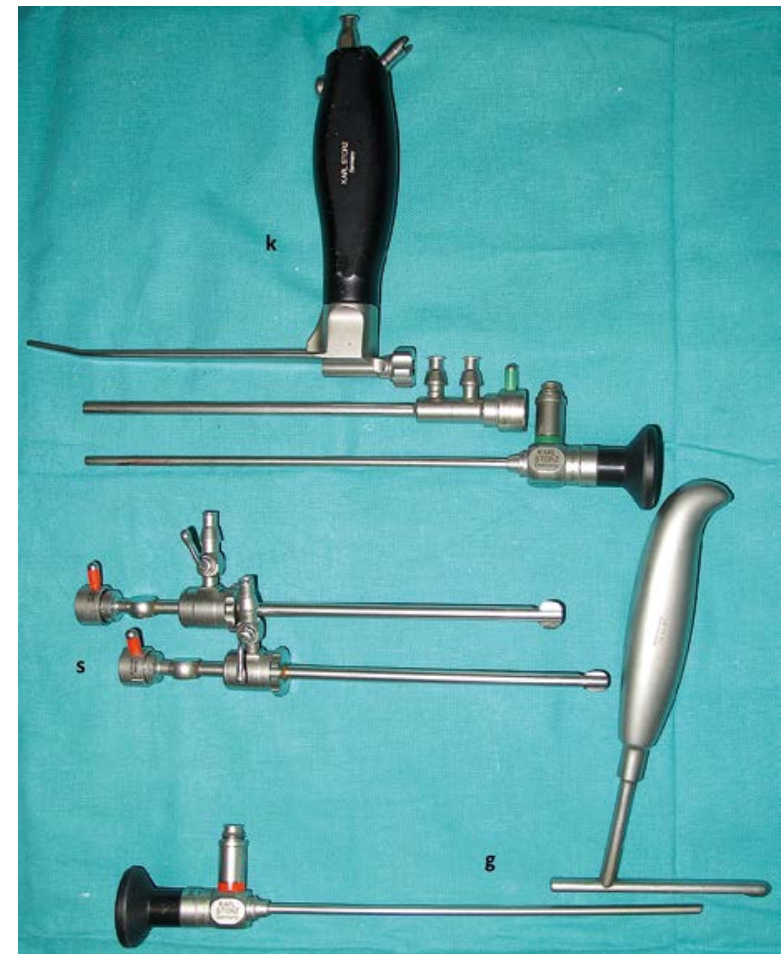

Photo 1. Three sets of instruments used for superficial endoscopic carpal tunnel release: (k) the Krishnan retractor with its irrigation sheath and $0^{\circ}$ endoscope, (s) narrow and wide endoscope sheaths with distal spatulae, and (g) the Medicon endoscope guide with the appropriate $30^{\circ}$ endoscope

should be taken to avoid inserting the guide inside the canal. Proximally, the guide reaches beyond the distal wrist crease. A $4 \mathrm{~mm}, 30^{\circ}$ endoscope fits into the guide's internal diameter (Photo 2). When inserted the endoscope allows one to view through the slit. It can be advanced or regressed inside the guide lumen, allowing a view of the TCL surface from above.

The Krishnan retractor is wider than the Medicon guide. It has a handle, and a tightening screw to allow for the attachment of a $0^{\circ}$ endoscope which is protected by an irrigation sheath. The retractor has a blunt blade on its end and this blade projects out further than the inserted endoscope to enable tissue retraction (Photo 1). This technique, however, requires the generation of a subcutaneous canal before the retractor can be inserted. A delicate needle holder should be introduced subcutaneously for this purpose. When the instrument is open and retracted, a narrow tunnel is created in the subcutaneous tissue. This allows for the safe introduction of the

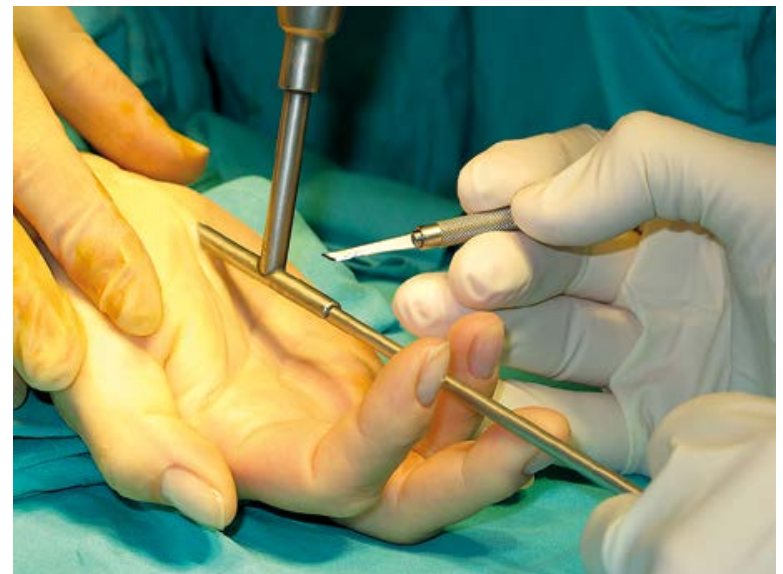

Photo 2. The Medicon guide with an inserted endoscope and surgical Micro blade. The superficial palmar portal approach is illustrated

retractor with its integrated endoscope. Care should be taken to avoid inserting the retractor inside the canal.

In order to safely transect the TCL, the median nerve should first be localized. To identify the position of the nerve, the skin should be lifted to reveal the distal end of the TCL. The ligament edge should then be delicately incised with a blade under direct observation and then the nerve can be localized. It enters under the palmar fat pad at the distal TCL edge. At this stage the retractor, or the guide and blade, are inserted contiguously to the external TCL surface. The ligament should be divided along the ulnar side of the median nerve. We use microsurgical blades (Micro blade mini \#15 Aesculap, B/Braun) secured in a thin handle (Photo 2). Although the thickened ligament may be transected using scissors, the use of a thin blade is probably less traumatic. The tight ligament splits easily under the blade, while the pliable epineurium bends, but remains unscratched (Photos $3 \mathrm{~A}$ and $3 \mathrm{~B}$ ). Scissors may be more useful in the distal forearm, as mentioned below. Palmaris brevis muscle fibres interposing the ligament fibres are frequently encountered during transection (Photo 4). They should also be divided, although care should be taken not to injure the thenar motor branch in its possible anomalous course.

In order to assist the viewing of anatomical details such as the anomalous motor branch, or to restrain fat which can occlude light, a third instrument, such as a retractor designed for endoscopic face lifting surgery, can be used. The endoscope sheath with a distal beak (spatula) for restraining tissue 

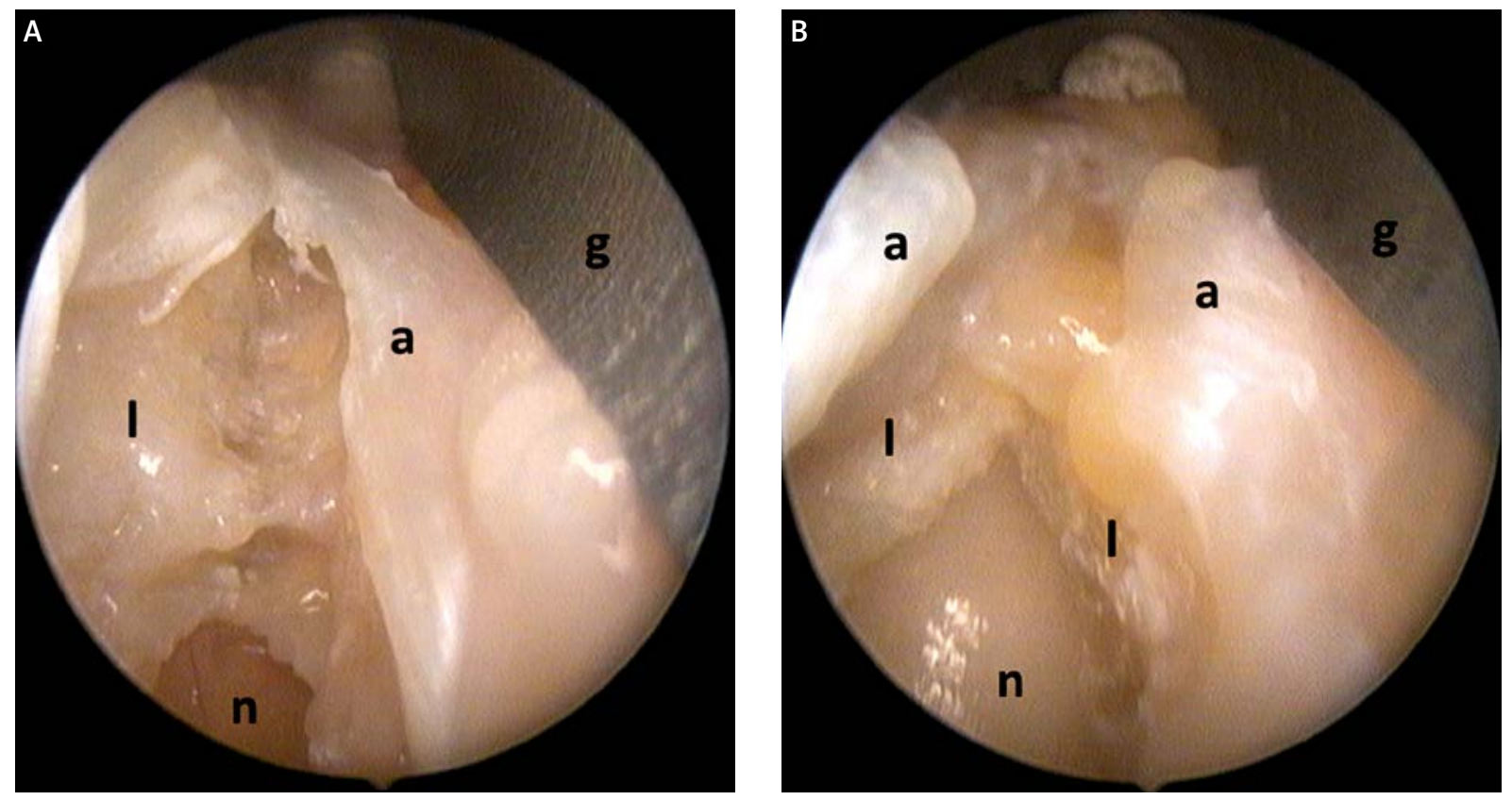

Photo 3. A - The superficial view for the partially incised carpal tunnel ligament through the slit in the Medicon guide. $\mathbf{B}$ - The view for an almost totally incised carpal tunnel ligament $n$-the median nerve, 1 - the ligament, a - the aponeurosis, $g$ - the Medicon guide

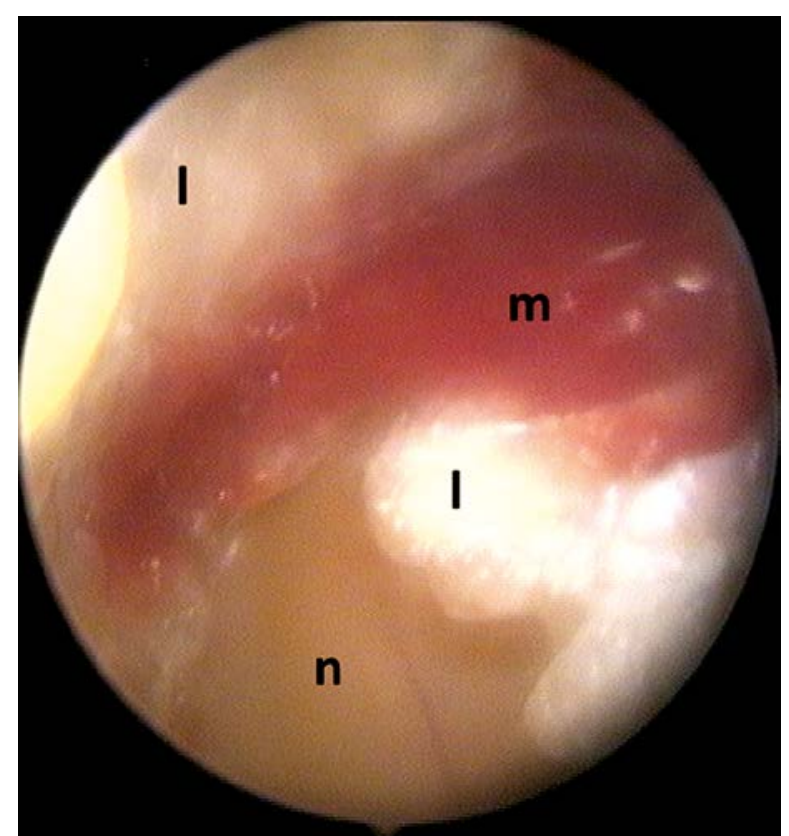

Photo 4. The view for the palmaris brevis muscle fibres interposing the ligament fibres $n$ - the median nerve, 1 - the ligament, $m$ - the muscle

(Karl Storz GmbH, Tuttlingen, Germany) is excellent for this purpose (Photo 1 ). It should be attached to the $0^{\circ}$ or $30^{\circ}$ endoscope instead of the Krishnan retractor and the regular irrigation sheath. Its spatu- la-like beak at the distal end enables its insertion inside the subcutaneous tissue, or under the superficial TCL layer, or between muscle and transverse ligament fibres.

\section{Discussion}

Superficial endoscopy for carpal tunnel release circumvents any unnecessary compression of the median nerve in the already constricted tunnel. Published reports of such an approach are, however, quite rare. Although the idea is similar to the endoscopic release of the cubital tunnel, this technique also resembles open mini-incision, device-assisted carpal tunnel release. Therefore, the subcutaneous endoscopic approach should, perhaps, be qualified as endoscope-assisted. Nevertheless, despite searching through the literature we only came across two such reports, which we will discuss below.

The use of the Medicon endoscope guide for the incision of the carpal ligament is illustrated in the Medicon catalogue for plastic surgery. Special knives designed for this technique are represented in this draft and are commonly available. These knives are similar to those proposed by Mirza [3]. His technique is, however, different since he inserted the endoscope inside the carpal tunnel without the guide. 

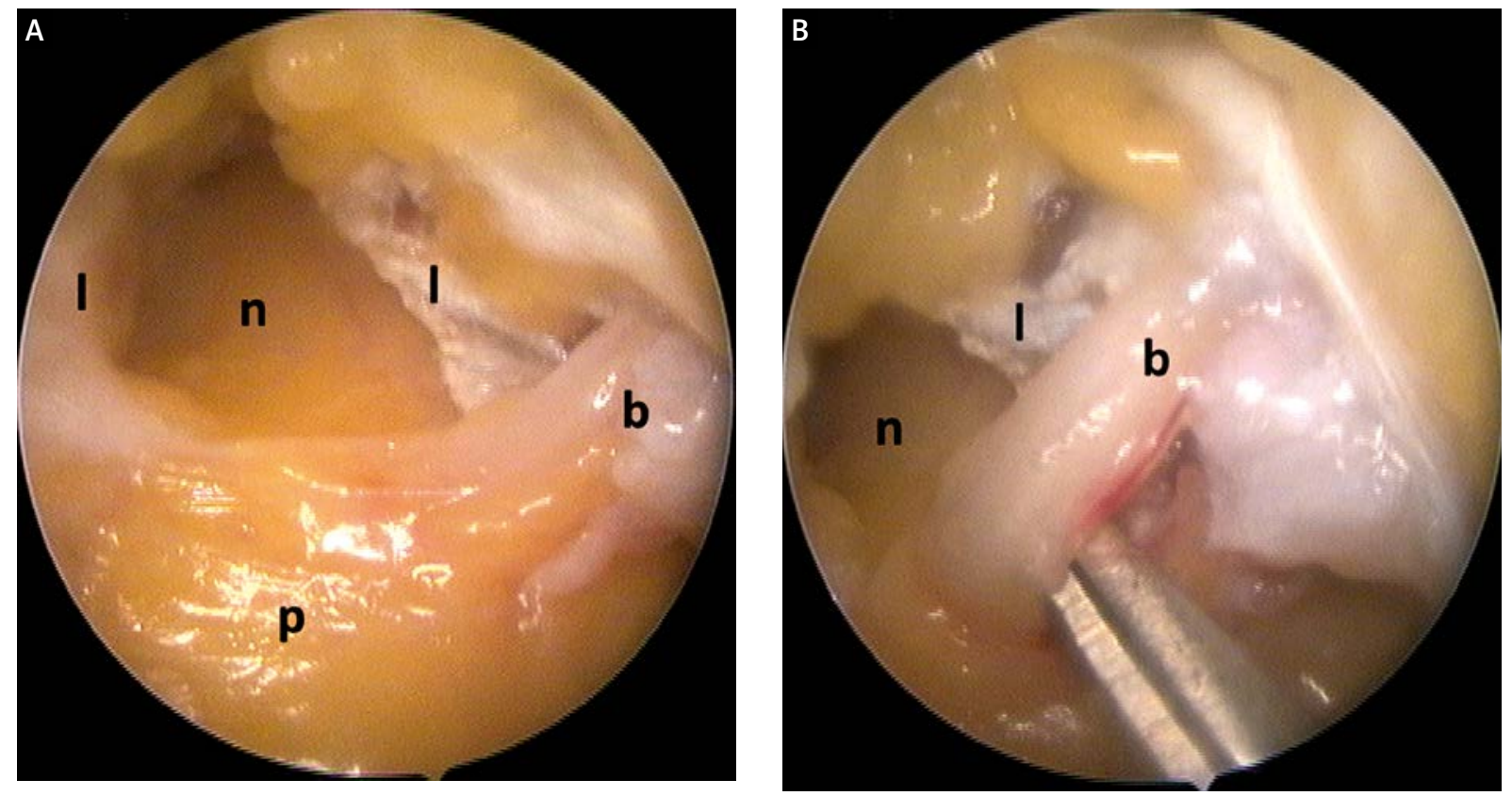

Photo 5. A - The view for the totally incised carpal tunnel ligament, the palmar fat pad and the motor branch of the median nerve. B - The close-up view for the motor branch of the median nerve (scissors indicate the nerve)

$n$-the median nerve, 1 - the ligament, $p$ - the palmar fat pad, $b$ - the motor branch

We were unable to find any other similar reports - neither the source, nor the author of Medicon instruments or techniques. The company information service also could not provide us with any adequate data.

The use of the Krishnan retractor has been described for single nerve entrapments, including the ulnar nerve and tarsal tunnel [4]. Doctor Krishnan is also the author of a brochure issued by Storz where his technique for endoscopic carpal tunnel release is presented. The author uses the proximal port and scissors to transect the TCL. From the proximal port and subcutaneous access he is able to view the nerve and assess it for any additional pathology [4]. In our experience, however, the proximal approach jeopardizes the distal nerve branches with the retractor blade when the palmar fat pad has been reached. We therefore think that the distal portal approach is more advantageous. This is accomplished through an incision distally from a modified Kaplan's line which represents a surface marker for the thenar motor branch of the median nerve [5]. Although presence of the branch may be confirmed at the beginning of the operation, we normally continue the dissection proximally. This nerve position should be determined if a variation of its course is suspected.

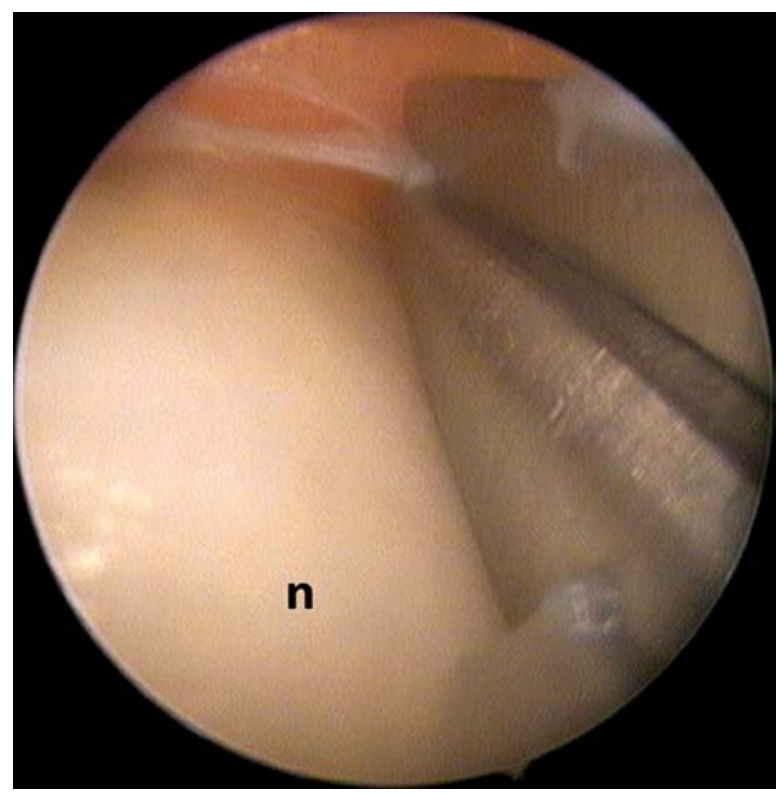

Photo 6. The view from the endoscope protected by the sheath with a distal spatula. The median nerve (n), and the scissors cutting the fascial band in the distal forearm can be seen

In cases of an aberrant palmaris brevis, or flexor pollicis brevis interposing the TCL fibres, the transligamentous pattern of this nerve is possible. In such 
cases the existence of the branch in its anatomical position should be verified (Photos $5 \mathrm{~A}$ and $5 \mathrm{~B}$ ). The distal incision facilitates its localization. If its anatomical position cannot be confirmed, the branch should be identified between the palmaris brevis fibres and protected before the entire TCL transection has been accomplished [6].

To facilitate the dissection between the muscle and the TCL fibres, the endoscope can be inserted into the irrigation sheath with the distal spatula. Although some authors have observed that the TCL fibre orientation is predominantly unidirectional and transverse [7], others have shown that the superficial layer of carpal ligament is longitudinal and in continuity with the antebrachial fascia and the aponeurosis [8]. The superficial ligament layer (Photo $3 \mathrm{~A}$ ), as well as the deeper fibre layers interlaced with muscle (Photo 4), are often visible during superficial endoscopy. The sheath distal spatula enables the separation of the fibres, facilitating attempts at controlled division. It is also convenient proximally in the distal forearm, where thickened antebrachial fascia may cover the median nerve several centimetres proximally from the TCL [9]. The endoscope sheath with a distal spatula may also be used as the sole instrument during the entire operation. This option may be indicated for obese patients where fat should be restrained with the spatula to enable visibility.

The use of a similar irrigation sheath with a larger distal retractor has been recently reported by Ip et al., for subcutaneous carpal tunnel release [10]. The main difference between this and our description is the proximal port and the use of scissors as proposed by the authors of this publication. We believe, however, that the use of scissors is more traumatic than a surgical blade. Cutting with scissors requires that one of the blades be inserted inside the narrow tunnel. When dissection begins in the distal port, it may be easily done only in the forearm, when the thick and firm ligament has already been cut with a surgical blade (Photo 6). The use of scissors from the proximal port jeopardizes the motor branch, when the palmar fat pad is reached. Ip et al. reported only minor complications such as pillar pain and hypertrophic scars; however, they only operated on ten patients [10]. The use of our technique coincided with two cases of palmar branch lacerations, but we operated on more than two hundred hands with the spatula endoscope sheath. This problem was discussed in our previous paper [2]. We believe that these complications were not caused by the use of the instrument, but rather were related to the limited visibility between the anomalous ligament fibres. There is always a risk of such complications regardless of the endoscopic technique used [11].

\section{Conclusions}

We have described three, rarely cited techniques for superficial endoscopic carpal tunnel release which in some aspects are advantageous over techniques where the endoscope is guided inside the constricted canal. The required instruments are inexpensive and easy to use for a surgeon experienced in arthroscopy.

\section{References}

1. DH, Paulson JC, Lane-Larsen CL, et al. Endoscopic carpal tunnel release: a comparison of two techniques with open release. Arthroscopy 1993; 9: 498-508.

2. Noszczyk BH, Nowak M, Krześniak N. Use of the accordion severity grading system for negative outcomes of carpal tunnel syndrome. J Plast Reconstr Aesthet Surg 2013; 66: 1123-30. .

3. Mirza MA, King ET Jr, Tanveer S. Palmar uniportalextrabursal endoscopic carpal tunnel release. Arthroscopy 1995; 11: 82-90.

4. Krishnan KG, Pinzer T, Schackert G. A novel endoscopic technique in treating single nerve entrapment syndromes with special attention to ulnar nerve transposition and tarsal tunnel release: clinical application. Neurosurgery 2006; 59 (Suppl. 1): ONS89-100.

5. Wilhelmi BJ, Mowlavi A, Neumeister MW, et al. Surface landmarks to locate the thenar branch of the median nerve: an anatomical study. Plast Reconstr Surg 2003; 111: 1612-5.

6. Green DP, Morgan JP. Correlation between muscle morphology of the transverse carpal ligament and branching pattern of the motor branch of the median nerve. J Hand Surg Am 2008; 33: 1505-11.

7. Prantil RK, Xiu K, Kim KE, et al. Fibre orientation of the transverse carpal ligament. Clin Anat 2012; 25: 478-82.

8. Stecco C, Macchi V, Lancerotto L, et al. Comparison of transverse carpal ligament and flexor retinaculum terminology for the wrist. J Hand Surg Am 2010; 35: 746-53.

9. Pacek CA, Chakan M, Goitz RJ, et al. Morphological analysis of the transverse carpal ligament. Hand (N Y) 2010; 5: 135-40.

10. Ip WY, Sweed TA, Fung KK, et al. A new technique of single portal endoscopic carpal tunnel release. Tech Hand Up Extrem Surg 2012; 16: 27-9.

11. Uchiyama S, Nakamura K, Itsubo T, et al. Technical difficulties and their prediction in 2-portal endoscopic carpal tunnel release for idiopathic carpal tunnel syndrome. Arthroscopy 2013; 29: 860-9.

Received: 14.08 .2013 , accepted: 14.10.2013 